\title{
Towards a new International System of Units (SI)
}

At a recent meeting of National Metrology Institute (NMI) Directors and representatives of Member States of the Convention of the Metre, the new chair of CIPM's (International Committee for Weights and Measures) Consultative Committee for Mass and Related Quantities, Philippe Richard, METAS (Switzerland) reported about the new roadmap of this committee towards the redefinition of the kilogram. This is a central issue for moving to a new International System of Units (SI). It is now planned to execute the various tasks, including the experiments, data evaluation, reviews and consultations sufficiently in advance so that the General Conference on Weights and Measures (CGPM) will be able to decide on the new SI in 2018.

Besides further experimental data from Watt balance measurements and studies on the silicon sphere (formerly called 'Avogadro' project), extraordinary calibration exercises with the current highest metrological artefact, the International Prototype Kilogram, have to be run. The Interdisciplinary Scientific Committee of the International Council for Science dealing with physical constants, CODATA, will also have to discuss and agree on the Planck constant derived from experiments with Watt balances as well as the silicon sphere measurements. Moreover, there are significant challenges to explain the redefinition and the traceability roots for the kilogram afterwards. It is envisaged that the new SI should bring more stability for the units. It will move the units for electricity back to the others (with uncertainty improvements for electricity measurements), but will not create changes of quantity values relevant for people and economy. 